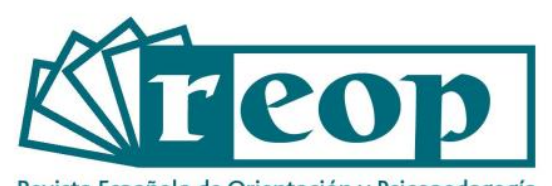

Revista Española de Orientación y Psicopedagogía

\title{
AUTOPERCEPCIÓN DE LAS COMPETENCIAS DE CREATIVIDAD E INNOVACIÓN EN ESTUDIANTES UNIVERSITARIOS EN CIENCIAS DE LA SALUD: FACTORES DE DESARROLLO
}

\section{SELF-PERCEPTION OF CREATIVITY AND INNOVATION COMPETENCES IN UNIVERSITY STUDENTS IN HEALTH SCIENCE: DEVELOPMENT FACTORS}

\author{
Pamela Marcone-Dapelo ${ }^{1}$ \\ Universidad de Playa Ancha. Facultad de Ciencias de la Salud. Departamento Disciplinario de \\ Kinesiología. Valparaíso, Chile \\ María Fernanda Agudelo Vizcaíno ${ }^{3}$ \\ Universidad de Playa Ancha. Facultad de Ciencias de la Salud. Departamento Disciplinario de \\ Fonoaudiología. Valparaíso, Chile \\ Maryelsa Rojas López ${ }^{4}$ \\ Universidad de Playa Ancha. Facultad de Ciencias de la Salud. Departamento Disciplinario de \\ Fonoaudiología. Valparaíso, Chile \\ Joselyn Godoy-Briceño ${ }^{5}$ \\ Universidad de Playa Ancha. Facultad de Ciencias de la Salud. Departamento Disciplinario de \\ Kinesiología. Valparaíso, Chile

\section{José González Campos 6}

Universidad de Playa Ancha. Facultad de Ciencias Naturales Exactas, Departamento Disciplinario de Matemática y Estadística. Valparaíso, Chile

${ }^{1}$ Correspondencia: Pamela Marcone-Dapelo Correo-e: pamela.marcone@upla.cl 


\section{RESUMEN}

Introducción: En los nuevos escenarios de educación universitaria, las competencias blandas son imprescindibles en la formación de grado de las carreras sanitarias, entre ellas están la creatividad e innovación, siendo pertinente indagar su comportamiento. Objetivo: Establecer el nivel de autopercepción de la creatividad e innovación que poseen los estudiantes que conforman la muestra, para determinar la orientación de dichas competencias y evidenciar si existe asociación estadísticamente significativa entre ellas y los factores intrínsecos (sexo y edad) y extrínsecos (ciclo de formación y carrera que cursa). Método: se utilizan técnicas cuantitativas descriptivas, mediante un diseño no experimental y transversal, en el que se recolectó la información mediante la adaptación chilena del "Cuestionario de Competencias Creativas e Innovadoras en Estudiantes Universitarios" $(3 \mathrm{Cl})$, a una muestra probabilística y estratificada $(\mathrm{N}=413)$, de la Facultad de Ciencias de la Salud de la Universidad de Playa Ancha. Para el análisis de la información se empleó el programa estadístico $R$ ( $R$ commander) y Jamovi. Resultados: el análisis factorial del instrumento establece cinco dimensiones, cuyos puntajes permitieron determinar el nivel de autopercepción de la creatividad e innovación y la orientación de ellos. El test de dependencia de Pearson, determina que solo existe asociación significativa ( $p$-valor 0.000 ) entre nivel y orientación de la creatividad e innovación, con la variable edad. Conclusiones: se aporta una adaptación para Chile del instrumento, la cual cumple estándares de fiabilidad y validez, proponiendo una alternativa para indagar la autopercepción del estado de estas competencias, en función de niveles de desarrollo y orientaciones.

Palabras clave: Creatividad; Innovación; Salud; Test de creatividad e innovación, Universidad.

\section{ABSTRACT}

Introduction: In the new context for higher education, soft skills, such as creativity and innovation, are paramount for health professionals. Thus, it is relevant to assess their behavior. Aim: To establish levels of creativity and innovation self-perception in a sample of students, and to evaluate if there is a statistically significant association among them and intrinsic (gender and age) and extrinsic factors (career major and progress in the academic program). Method: A cross-sectional, non-experimental design is used. Data was collected using the Chilean adaptation of the "Cuestionario de Competencias Creativas e Innovadoras 3Cl", which was filled out by a stratified probabilistic sample of 413 students of a health sciences faculty. The software programs $R$ and Jamovi were used for statistical analysis. Results: Factorial analysis of the questionnaire yields 5 dimensions, the scores of which allowed to characterize the sample regarding levels of selfperception in creativity and innovation. Pearson correlation coefficient indicates that the only significant association $(\mathrm{p}<0.01)$ was between the level and orientation of creativity-innovation, and age. Conclusions: This study contributes with an adaptation for Chile of the original questionnaire, which meets the standards of reliability and validity, proposing an alternative to investigate the state of these competences, based on levels of development and direction.

Key Words: Creativity; innovation; health; creativity and innovation questionnaire; University

\section{Cómo citar este artículo:}

Marcone-Dapelo, P., Agudelo, M., Rojas, M., Godoy-Briceño, J. y González, J. (2020). Autopercepción de las Competencias de Creatividad e Innovación en estudiantes Universitarios en Ciencias de la Salud: Factores de Desarrollo. Revista Española de Orientación y Psicopedagogía, 31(3), 64-85. https://doi.org/10.5944/reop.vol.31.num.3.2020.29262 
Autopercepción de las competencias de creatividad e innovación... Pamela Marcone-Dapelo et al.

\section{Introducción}

La formación de grado debe asegurar el desarrollo integral del estudiantado (Jiménez et al., 2013), creando condiciones en los individuos para ampliar sus posibilidades de obtener empleo en este entorno global y dinámico (Trujillo-Segoviano, 2014), las organizaciones requerirán profesionales cada vez más creativos que logren convertir sus ideas en riqueza para la sociedad (Arias et al., 2013).

Las universidades tienen como desafío, adaptarse a nuevas circunstancias, establecer procesos de creación e innovación, generar capacidades para acceder al conocimiento, junto a una actitud de análisis y comprensión de la dinámica social actual (Arias et al., 2013). La creatividad depende de tres factores significantes en educación: igualdad, calidad e implementación de reformas educativas modernas (Pllana, 2019); favoreciendo la formación de profesionales con un alto valor de humanidad y compromiso social (Paz et al., 2016) elevando su capacidad de reflexión creativa, para la evaluación crítica, resolución de problemas, toma decisiones y adaptarse a un mundo cambiante (Valera, 2010).

En el caso de estudios universitarios en salud, no solo se necesita un mayor conocimiento teórico, sino la capacidad de darle variabilidad a su saber; para ello se reconoce a la creatividad e innovación como pilares fundamentales en el desarrollo integral de la profesión (Marcone-Dapelo et al., 2018), haciéndose necesario contar con un instrumento adaptado a la educación universitaria chilena, que permita describir y caracterizar la autopercepción de competencias de creatividad e innovación en estudios universitarios en ciencias de la salud.

Por ello, el objetivo del presente estudio es establecer el nivel de autopercepción de la creatividad e innovación que poseen los estudiantes de la Facultad de Ciencias de la Salud (FCS) de la Universidad de Playa Ancha (UPLA), para determinar la orientación de dichas competencias y evidenciar si existe asociación estadísticamente significativa entre ellas y los factores intrínsecos y extrínsecos de los sujetos de la muestra.

\section{Antecedentes Teóricos}

Las competencias responden a procesos complejos, donde los individuos actúan de manera creativa ante problemas cotidianos con el fin de darles solución; integrando el saber ser, saber conocer y saber hacer (Trujillo-Segoviano, 2014); la competencia moviliza los recursos para la resolución de problemas complejos (Jiménez et al., 2014). Existen dos tipos de competencias, duras y blandas, de manera de diferenciar las habilidades técnicas de las interpersonales, siendo las segundas, habilidades personales que corresponden sobre todo al terreno cognitivo, del pensamiento y al ámbito social (Neri et al., 2019). Las competencias blandas se requieren actualmente por parte de organizaciones en un entorno global; siendo la creatividad una de ellas (Neri et al., 2019; Rueda, 2020).

En el área de salud es imperioso contar con un modelo pedagógico fortalecido por un aprendizaje significativo y continuo, que se desarrolla de forma dinámica, durante su vida profesional (Pinilla, 2018). En este enfoque los estudiantes se comprometen con sus actividades 
de aprendizaje (Chen et al., 2018), asumiendo un papel consciente en la resolución de problemas reales (Pinilla-Roa, 2012). Desde la metodología de enseñanza - aprendizaje, el desarrollo de la competencia creatividad e innovación (Arias et al., 2013) es imprescindible para todo profesional (Hernández et al., 2015).

La creatividad es la capacidad que tiene el ser humano de pensamiento divergente, favorece la búsqueda de soluciones frente a problemas, y la innovación es la capacidad de implementar dichas ideas de un modo diferente (Hernández et al., 2015; Bermejo et al., 2010). Además, se considera una capacidad de carácter cognitivo-afectivo que le permite a la persona organizar el proceso psicológico que lo lleve a mostrar un comportamiento nuevo u original, flexible, fluido y organizado, orientado a la búsqueda, la detección y solución de un problema (Velásquez et al., 2010). Así, la creatividad no se produce en el interior de las personas sino, más bien, en la interacción entre éstas, su pensamiento y el contexto sociocultural en el que se encuentran (Fernández et al., 2012). Esta competencia puede ser desarrollada en graduaciones variables por cada persona en función de una serie de circunstancias (Tena, 2010), a esto contribuye, las características personales del sujeto y la función de los atributos creativos personales, incluyéndose aquí dimensiones de naturaleza cognitiva (fluidez o flexibilidad de pensamiento) y factores no cognitivos (personales y sociales, como tolerancia a la ambigüedad, propensión a asumir riesgos, motivación intrínseca, autoeficacia o los estilos creativos (Oliveira et al., 2009).

Lo anterior ha llevado a la creación de instrumentos que midan la autopercepción por parte de los estudiantes de su nivel de creatividad e innovación, es así como el $3 \mathrm{Cl}$, "cumple satisfactoriamente con la función de diagnóstico de las competencias creativas e innovadoras" (p.144), pero como plantean las autoras, aún se encuentra en fase piloto, siendo necesario realizar un muestreo con mayor cantidad de participantes (Iglesias-Cortiza et al., 2013).

En el escenario del nuevo enfoque en la formación profesional, la FCS de la UPLA, en su modelo educativo, promueve la innovación curricular de los estudios en ciencias de la salud que imparte. La formación disciplinar se sistematiza a través de tres ciclos formativos, de acuerdo al nivel de desarrollo de las competencias declaradas en los perfiles profesionales: bachillerato, licenciatura y síntesis profesional (UPLA, 2017).

Siendo la creatividad e innovación competencias fundamentales en el proceso de formación de los estudiantes universitarios, es imprescindible conocer las dimensiones que la constituyen. Iglesias-Cortizas et al. (2013) señalan que existen 4 dimensiones de la creatividad: 1) actitud creativa, referida al autor de una idea (individuo), se encuentra conformada por la triada instinto, motivación e iniciativa para una tarea; 2) proceso creativo, donde el autor detecta un problema y genera una nueva idea para solucionarlo mediante acciones nuevas, no convencionales; 3) resultados creativos, esta es la acción y el efecto de conseguir algo innovador que se pueda aplicar en una tarea; y 4) sociocultural, que supone a la creatividad como un producto de interacción entre la persona y su contexto sociocultural.

Por su parte, Steers (2009) indica que Dewulf y Baillie identifican cuatro elementos en el proceso de creatividad: 1) preparación, en el cual se define el problema, para reformularlo y redefinirlo, dándole un entendimiento; 2) generación, en el que se va más allá de las vías habituales del pensamiento, existe una depuración asociativa y conceptualización del problema; 3) incubación: en la que se encuentra un estímulo subconsciente, a menudo luego de un periodo de relajación; y 4) verificación, en la que se analizan las ideas, agrupando, evaluando y posteriormente, planeando.

La creatividad e innovación presentan niveles de desarrollo, situando al individuo en estadios determinados, que en el caso de educación universitaria podrían favorecer o ir en desmedro de su proceso de aprendizaje y nivel académico. Arias et al. (2013) plantean los niveles de desarrollo que se aprecian en la Tabla 1: 
Autopercepción de las competencias de creatividad e innovación... Pamela Marcone-Dapelo et al.

\section{Tabla 1}

Nivel de desarrollo de las competencias de creatividad e innovación

\begin{tabular}{cl}
\hline Nivel de desarrollo & \multicolumn{1}{c}{ Descripción } \\
\hline $\mathbf{1}$ & $\begin{array}{l}\text { Fortalecimiento y mantenimiento de la habilidad de creatividad e } \\
\text { innovación. }\end{array}$ \\
\hline $\mathbf{2}$ & $\begin{array}{l}\text { Cuenta con la competencia de creatividad e innovación, y la aplica en } \\
\text { diferentes ámbitos, pero presenta a mejorar. }\end{array}$ \\
\hline $\mathbf{3}$ & $\begin{array}{l}\text { Utiliza sólo en ciertos momentos la competencia de creatividad e } \\
\text { innovación o cuando se le pide que haga uso de ella }\end{array}$ \\
\hline $\mathbf{4}$ & $\begin{array}{l}\text { No tiene como prioridad el desarrollo de la competencia de creatividad e } \\
\text { innovación. }\end{array}$ \\
\hline $\mathbf{5}$ & No cuenta con la competencia de innovación y creatividad.
\end{tabular}

Fuente: Arias et al. (2013)

Los autores destacan que quienes se encuentran en los puntos 1 y 2 evidencian un proceso de fortalecimiento y expansión de la competencia. Sin embargo, si se encuentran entre el nivel 3 y 5 , es necesario desarrollar y generar la competencia en las diversas dimensiones (Arias et al., 2013). Asimismo, el nivel de desarrollo de dichas competencias también depende del sexo y la edad. Caballero et al. (2019) identificaron que las mujeres estudiantes universitarias tienden a ser más creativas, mientras que, Caballero et al. (2018) al evaluar a estudiantes de secundaria, no encontraron diferencias significativas entre ellos. En relación a la edad, Caballero et al. (2019) indican que los alumnos menores de 20 años son los que destacan con los mayores niveles de creatividad.

\section{Método}

Investigación con enfoque cuantitativo, de tipo reporte de investigación empírica descriptivacorrelacional, con un diseño no experimental y transversal (Hernández et al., 2014). Se recolectó la información mediante la adaptación chilena del "Cuestionario de Competencias Creativas e Innovadoras en Estudiantes Universitarios" (3Cl), elaborado en España y validado en una muestra menor en Chile, el cual cumple con tener todas las características psicométricas deseadas. La información recabada se sometió a análisis a través del programa estadístico $R$-commander (open source), destacado por su potencia de análisis y la programación de estadísticas específicas, complementariamente, por la calidad gráfica se utilizó Jamoví (open source).

\section{Objetivo}

Establecer el nivel de autopercepción de la creatividad e innovación que poseen los estudiantes de la Facultad de Ciencias de la Salud (FCS) de la Universidad de Playa Ancha (UPLA), para determinar la orientación de dichas competencias y evidenciar si existe asociación estadísticamente significativa entre ellas y los factores intrínsecos y extrínsecos de los sujetos de la muestra. 
Autopercepción de las competencias de creatividad e innovación... Pamela Marcone-Dapelo et al.

\section{Hipótesis:}

- Existen diferencias estadísticamente significativas ( $p$-valor $\leq 0,05)$ entre las dimensiones consultadas en la adaptación chilena del $3 \mathrm{Cl}$.

- Existe asociación significativa ( $p$-valor $\leq 0,05$ ) en función del nivel y la orientación de la creatividad e innovación, respecto de factores intrínsecos de la muestra

- Existe asociación significativa ( $p$-valor $\leq 0,05$ ) en función del nivel y la orientación de la creatividad e innovación, respecto de factores extrínsecos de la muestra.

\section{Muestra}

La población del presente estudio está conformada por la totalidad de estudiantes de la FCS $\mathrm{N}=1390$. Compuesta por las disciplinas de kinesiología, terapia ocupacional, nutrición y dietética, enfermería y fonoaudiología de la UPLA.

La muestra es de tipo probabilística, con muestreo estratificado, considerando cada carrera como un estrato. La muestra está constituida por un total de 413 estudiantes de las cinco carreras de la FCS, de diferentes cohortes, la cual es equivalente al $30 \%$ de la población, superando en 111 unidades muestrales lo sugerido por la fórmula de Namakforoosh (2000), considerando un nivel de significancia del $5 \%$ y un error de estimación del $5 \%$. Del total de la muestra, 308 sujetos son mujeres $(74,6 \%)$ y 105 hombres $(25,4 \%)$, fluctuando las edades entre los 18 y 38 años.

Los respectivos tamaños muestrales según estrato se describen en la siguiente Tabla:

\section{Tabla 2}

Organización de estratos de la muestra

\begin{tabular}{lcc}
\hline Estratos & Tamaño Muestrales & Porcentaje \\
\hline Terapia Ocupacional & 70 & $20 \%$ \\
\hline Fonoaudiología & 82 & $41 \%$ \\
\hline Kinesiología & 132 & $43,14 \%$ \\
\hline Nutrición y Dietética & 64 & $24,62 \%$ \\
\hline Enfermería & 65 & $23,72 \%$ \\
\hline
\end{tabular}

Fuente: Elaboración propia

La distribución de los estudiantes de la FCS acorde a los ciclos de formación es la siguiente: "Ciclo Inicial" 198 (45\%), "Ciclo Intermedio" 186 (45\%) y “Ciclo de Síntesis Profesional” 29 (7\%). 
Autopercepción de las competencias de creatividad e innovación... Pamela Marcone-Dapelo et al.

Instrumentos y/o técnicas

Instrumento "Cuestionario de Competencias Creativas e Innovadoras en Estudiantes Universitarios" (3Cl)

El $3 \mathrm{Cl}$ es un instrumento cuyo objetivo es establecer la autopercepción de las competencias de creatividad e innovación de estudiantes universitarios españoles (Iglesias-Cortizas et al, 2013). El instrumento consta de 33 ítems, los cuales se puntúan mediante una escala tipo Likert, en la que se valora desde "Nada o Nunca" <1> a "Mucho o Siempre" $<4>$.

El análisis factorial del instrumento original genera 10 factores que explican el $63 \%$ de la interrelación entre variables, con un $\mathrm{N}=131$ (Iglesias-Cortizas et al., 2013). Posteriormente se realizó un estudio piloto que pretendía aportar una adaptación del instrumento a la población chilena, arrojando una matriz de componentes principales que confirma la estructura factorial con un $66,79 \%$ de la interrelación; de la misma forma, en post de explorar el comportamiento psicométrico del instrumento, en consideración a lo reducido de las muestras estudiadas, se lleva a cabo una segunda investigación con un $\mathrm{N}=282$ sujetos, de la que se obtiene una estructura factorial similar a la de los estudios piloto previamente realizados en España y Chile (MarconeDapelo et al., 2018).

Con base en los estudios previos, se hace necesario continuar con la línea de investigación, pero contando con un $\mathrm{N}$ mayor, amplia cobertura de disciplinas del ámbito de la salud y con un diseño metodológico probabilístico.

\section{Procedimiento y Criterios de Rigor}

Se ha utilizado como criterio de decisión la estadística p-valor, considerando un nivel de significancia de 5\%. Como estimador de la fiabilidad se utilizará el coeficiente Alfa de Cronbach. En relación a la validez se utilizará como criterio de estimación el índice de validez Gamma (González et al., 2016).

De manera confirmatoria se desarrollará un análisis factorial, con rotación varimax y estadística de prueba Chi cuadrado, para establecer si la varianza explicada por los factores es significativa.

Previo a la aplicación del instrumento diseñado cada estudiante firma voluntariamente un consentimiento informado. La administración del instrumento es realizada por los investigadores en las aulas de clase durante el segundo semestre académico del año 2018.

La información recolectada es resguardada mediante codificación y almacenaje restringido, acorde con lo estipulado en el artículo 29 de la Ley 17.374 del secreto estadístico.

\section{Variables}

El presente estudio contempla variables independientes y dependientes. Dentro de las primeras se consideraron aquellas inherentes al individuo, como son sexo y edad, las que se catalogarán como variables intrínsecas. Mientras que aquellas que involucran aspectos de orden académico, 
Autopercepción de las competencias de creatividad e innovación... Pamela Marcone-Dapelo et al.

como son disciplina de estudio profesional y ciclo formativo que cursa, se entenderán como variables extrínsecas.

Variables Dependientes:

- Innovación:

- Definición conceptual: destreza para generar y adaptar ideas creativas coherentes con contextos específicos (Iglesias-Cortizas et al., 2013).

- Creatividad:

- Definición conceptual: capacidad de formular y resolver problemas de manera divergente, original y alternativa, integrando conocimientos existentes con nuevas perspectivas (Chiecher et al., 2018).

- Definición Operacional de creatividad e innovación: corresponde a la media aritmética obtenida a partir de la sumatoria de las puntuaciones brutas. Las cuales se calculan para el puntaje total y dimensiones del instrumento, generando niveles de desarrollo de la creatividad e innovación y orientaciones que marcan las tendencias del enfoque de la creatividad e innovación.

- Niveles de creatividad e innovación:

- Estadio Experiencial: Conducta creativa basada en el desarrollo del individuo y sus experiencias con el entorno.

- Estadio Asociativo: Actitud del individuo de adaptar su creatividad a situaciones diversas, para encontrar posibles soluciones.

- Estadio Productivo: Capacidad para aplicar las ideas novedosas, en función de resolver una problemática.

- Estadio Social: Habilidad para crear una idea que contribuya más que a sí mismo, a un grupo social.

- Estadio Innovador: Destreza para transformar las ideas en productos novedosos y válidos a nivel sociocultural en un momento determinado.

- Orientaciones de creatividad e innovación:

- Proactiva: es el comportamiento de un sujeto caracterizado por ser orientado al cambio y que se anticipa de forma voluntaria a una situación futura.

- Analítica: comportamiento de un sujeto que examina en detalle el entorno para obtener información de él y contribuir en la toma de decisiones.

- Planificadora: comportamiento de un sujeto que genera un sistema de organización, basado en una estrategia para abordar una situación determinada.

- Reguladora: comportamiento de un sujeto que mide y ajusta su actuar, en concordancia con las características de la situación.

- Progresista: comportamiento de un sujeto caracterizado por la generación de ideas avanzadas que contribuyen a la mejora de sí mismo y/o del entorno biopsicosocial. 
Autopercepción de las competencias de creatividad e innovación... Pamela Marcone-Dapelo et al.

Variables Independientes:

Intrínsecas:

- Sexo:

- Definición operacional: Variable de tipo dicotómica, cuyos estados son Femenino Masculino.

- Edad:

- Definición operacional: Variable cuantitativa continua, en escala de razón. Para procurar un análisis más comprensible, se establecen rangos etarios, estipulando cinco clases; las cuales se generaron considerando un eje de simetría y propendiendo a no polarizar, quedando distribuidos de la siguiente forma:

- Rango 1 de [18; 22], con una frecuencia de 238 casos equivalente al $57.63 \%$ de la muestra;

- Rango 2 de [22; 26], con 141 sujetos equivalentes al $34.14 \%$;

- $\quad$ Rango 3 de [26; 30], con 27 casos equivalentes al 6.53\% muestral;

- $\quad$ Rango 4 de [30; 34], con 5 sujetos equivalente al 1,2\% de la unidad muestral

- $\quad$ Rango 5 de $[34 ; 38]$ con 2 estudiantes equivalente al $0.5 \%$.

\section{Extrínsecas:}

- Estudio profesional:

- Definición conceptual: Programa formativo perteneciente al nivel de educación universitaria, el cual habilita a la persona que lo cursa y aprueba en una determinada disciplina.

- Definición operacional: Variable cualitativa en escala nominal, cuyos estados son:

- Kinesiología

- Terapia Ocupacional.

- Nutrición y Dietética.

- Enfermería.

- Fonoaudiología.

- Ciclo formativo:

- Definición conceptual: Segmento de un plan de estudios en que se consideran hitos para el desarrollo de ciertos niveles de competencias.

- Definición operacional: Variable cualitativa en escala ordinal, cuyos estados se describen:

Ciclo 1 - Bachillerato: período que abarca desde el primer al cuarto semestre del plan de estudios. 
Autopercepción de las competencias de creatividad e innovación... Pamela Marcone-Dapelo et al.

Ciclo 2 - Licenciatura: período que abarca desde el quinto y hasta el octavo semestre del plan de estudios.

Ciclo 3 - Síntesis Profesional: Abarca al noveno y décimo semestre del plan de estudios.

\section{Resultados}

\section{Análisis Factorial}

Como estimador de la fiabilidad, particularmente utilizando Alfa de Cronbach se obtiene 0.9022 , reflejando una alta consistencia interna. La estimación de validez fue de 0,9024 (González et al., 2016).

En el análisis factorial se establecen cinco factores (rotación varimax, la estadística chi cuadrado fue de 721.94, con 373 grados de libertad y p-valor igual a 0,000), los que caracterizan las dimensiones "Actitud Proactiva", "Reconocimiento Propio y Externo", "Planificación Estratégica", "Regulación del Autoconcepto" y "Perspectiva al Cambio". Alcanzando un 40\% de varianza explicada, siendo esta significativa.

\section{Tabla 3}

Peso factorial según Matriz de componentes Rotados

\begin{tabular}{|c|c|c|}
\hline Factores & Componentes & Peso \\
\hline \multirow[t]{12}{*}{ Factor 1} & 18: "Cuando comienzo algo nuevo tengo muchas ideas para aplicar" & 0.232 \\
\hline & I14: "Tengo ideas nuevas para resolver los problemas" & 0.421 \\
\hline & I15: "El objetivo de crear cosas nuevas es cambiar el entorno físico" & 0.329 \\
\hline & I16: "Mi creatividad ayuda al cambio social" & 0.590 \\
\hline & $\begin{array}{l}\text { I23: "Soy capaz de detectar los problemas y generar soluciones } \\
\text { innovadoras" }\end{array}$ & 0.482 \\
\hline & $\begin{array}{l}\text { 124: "Me gusta proponer nuevas ideas innovadoras para los trabajos } \\
\text { de las asignaturas" }\end{array}$ & 0.593 \\
\hline & 125: "Cuestiono las ideas de siempre para innovar otras más eficaces" & 0.540 \\
\hline & $\begin{array}{l}\text { I26: "Evalúo las ideas innovadoras que se me presentan en el contexto } \\
\text { Universitario" }\end{array}$ & 0.565 \\
\hline & I28: "Estimulo el desarrollo de las capacidades de otros para innovar" & 0.433 \\
\hline & 129: "Siento un compromiso ético con generar innovación de calidad" & 0.573 \\
\hline & I30: "En general, soy una persona creativa" & 0.559 \\
\hline & I31: "En general, soy una persona innovadora" & 0.600 \\
\hline \multirow[t]{2}{*}{ Factor 2} & $\begin{array}{l}\text { 17: "Mi intuición es muy efectiva en el estudio y al desarrollar trabajos } \\
\text { de las asignaturas de la Universidad" }\end{array}$ & 0.278 \\
\hline & $\begin{array}{l}\text { 19: "Soy muy perseverante en el estudio al desarrollar trabajos de las } \\
\text { asignaturas de la Universidad" }\end{array}$ & 0.380 \\
\hline
\end{tabular}

Fuente: Elaboración propia 
Autopercepción de las competencias de creatividad e innovación... Pamela Marcone-Dapelo et al.

Tabla 3 (Continuación)

Peso factorial según Matriz de componentes Rotados

Factores

Componentes

Peso

Factor 2

110: "Auto-gestiono todas las actividades de mis trabajos y estudios

0.408

Universitarios"

118: "Valoro la originalidad del producto creado o innovado"

0.444

119: "Valoro la autonomía para mi creatividad e innovación"

0.505

120: "Valoro la crítica constructiva para la creatividad e innovación" $\quad 0.519$

121: "Valoro la capacidad de tomar decisiones"

122: "Valoro la planificación y organización de mi trabajo como 0.542 estudiante Universitario"

127: "Adopto las capacidades e ideas innovadoras de otros si éstas son $\quad 0.387$ eficaces"

Factor 3

I1: "Me gusta ampliar mis estrategias de estudio y de trabajo en la

0.825 Universidad"

I2: "Me estimula utilizar nuevos métodos de estudio y de trabajo en la Universidad"

13: "Procuro aplicar nuevas tecnologías cuando estudio y trabajo en las asignaturas de la Universidad"

14: "Elaboro nuevas estrategias que faciliten mi trabajo como 0.471 estudiante universitario"

15: "Procuro buscar nuevos desafíos para desarrollar mi trabajo en las asignaturas de la Universidad"

16: "Busco más conocimientos cuando estudio o desarrollo trabajos de $\quad 0.379$ las asignaturas de la Universidad".

$\begin{array}{lll}\text { Factor } 4 & \text { I11: "Mi autoestima me ayuda a crear cosas nuevas" } & 0.692\end{array}$

112: "Me auto-motivo para crear cosas nuevas"

0.716

113: "Me auto-controlo cuando las cosas no salen bien" $\quad 0.410$

Factor $5 \quad 117$ : "Creo que las tecnologías facilitan la innovación" $\quad 0.259$

132: "Considero que la creatividad e innovación pueden cambiar la 0.788 sociedad"

I33: "Considero que la creatividad e innovación pueden cambiar a la $\quad 0.683$ persona"

Fuente: Elaboración propia

\section{Tabla 4}

Organización de dimensiones por ítems del instrumento

\begin{tabular}{|c|c|c|c|}
\hline Factor & Nominación Nueva Dimensión & Ítem del instrumento & Dimensión original \\
\hline \multirow[t]{4}{*}{1} & \multirow[t]{4}{*}{ Actitud Proactiva } & 14,30 у 31 & 1 \\
\hline & & 15. & 2 \\
\hline & & $8,23,24,25,26$ у 28. & 3 \\
\hline & & 16 y29. & 4 \\
\hline \multirow[t]{3}{*}{2} & \multirow[t]{3}{*}{ Reconocimiento Propio y Externo } & 7,9 у 10 & 1 \\
\hline & & 18. & 2 \\
\hline & & $19,20,21,22$ у 27. & 3 \\
\hline \multirow[t]{2}{*}{3} & \multirow[t]{2}{*}{ Planificación Estratégica } & $1,2,5$ у 6 & 1 \\
\hline & & 3 у 4. & 2 \\
\hline 4 & Regulación del Autoconcepto & 11,12 y 13 & 1 \\
\hline 5 & Perspectiva al Cambio & 32,33 у 17. & 4 \\
\hline
\end{tabular}

Fuente: Elaboración propia

REOP. Vol. 31, no, 3er Cuatrimestre, 2020, pp. 64 - 85 [ISSN electrónico: 1989-7448] 


\section{Estadísticos Descriptivos por Dimensión}

Desde el punto de vista de la centralidad, particularmente basado en la media, es posible establecer la jerarquía siguiente: D5, D2, D3, D4, D1. Siendo la D1 la dimensión con menor puntuación y D5 la de mayor puntuación. Se debe indicar que esta jerarquización es basada en descriptivos, por tanto, en las secciones siguientes se establecerá si las diferencias observadas se deben a una causa (pruebas de significancia) o son simplemente diferencias por aleatoriedad.

\section{Tabla 5}

Resúmenes descriptivos por dimensión

\begin{tabular}{lccccr}
\hline & $\begin{array}{c}\text { Puntaje } \\
\text { Dimensión 1 }\end{array}$ & $\begin{array}{c}\text { Puntaje } \\
\text { Dimensión 2 }\end{array}$ & $\begin{array}{c}\text { Puntaje } \\
\text { Dimensión 3 }\end{array}$ & $\begin{array}{c}\text { Puntaje } \\
\text { Dimensión 4 }\end{array}$ & $\begin{array}{c}\text { Puntaje } \\
\text { Dimensión 5 }\end{array}$ \\
\hline Media & 2.82 & 3.20 & 2.92 & 2.88 & 3.49 \\
\hline Mediana & 2.83 & 3.22 & 3.00 & 3.00 & 3.67 \\
\hline $\begin{array}{l}\text { Desviación } \\
\text { Estándar }\end{array}$ & 0.511 & 0.438 & 0.524 & 0.691 & 0.504 \\
\hline Asimetría & -0.108 & -0.625 & -0.200 & -0.396 & -0.856 \\
\hline Curtosis & 1.21 & 0.503 & -0.0197 & -0.171 & 0.107 \\
\hline
\end{tabular}

Fuente: Elaboración propia

Por otro lado, y de manera complementaria, en términos de variabilidad y particularmente utilizando la desviación estándar, la Dimensión 4 es la de mayor heterogeneidad, sin embargo, en términos porcentuales no supera el $30 \%$.

En función de la mediana (percentil 50), no se observan grandes diferencias con respecto a la media, por lo tanto, se descarta la presencia de niveles atípicos.

En relación a la tendencia de los datos, no existen diferencias, siendo estos asimétricos negativos, esto significa que los datos tienden a agruparse en torno a valores altos de creatividad para cada dimensión. En cuanto a curtosis, las dimensiones 1 y 2 presentan un alto grado de concentración en torno a la media, siendo la distribución caracterizada como leptocúrtica. Mientras que las dimensiones 3, 4 y 5 son mesocúrticas (Ver gráficos de Densidades en Figura 1).

\section{Diferencias Significativas por Dimensión: Comparación inferencial por dimensión}

Con el fin de establecer el tipo de análisis inferencial a realizar, se desarrolló el Test de Normalidad de Shapiro Wilk para las variables en cuestión, considerando un nivel de significancia del $5 \%$ y tomando como criterio de decisión el $p$-valor.

En esta etapa los datos soportan evidencia a favor de la hipótesis alternativa, por lo tanto, los datos que caracterizan a cada una de las dimensiones no obedecen a la distribución normal, en consecuencia, los análisis se desarrollarán con pruebas no paramétricas, particularmente Wilcoxon $(\mathrm{W}<0,986$, $\mathrm{p}$-valor menor 0,000$)$.

En función de la jerarquía obtenida en el análisis descriptivo, las comparaciones que se realizarán son las siguientes: D5-D2, D2-D3, D3-D4, D4-D1, cuyos resultados son resumidos en el diagrama jerárquico siguiente: 
Autopercepción de las competencias de creatividad e innovación... Pamela Marcone-Dapelo et al.

\section{Figura 1}

Estimaciones de las funciones de densidad y sus respectivos box-plot

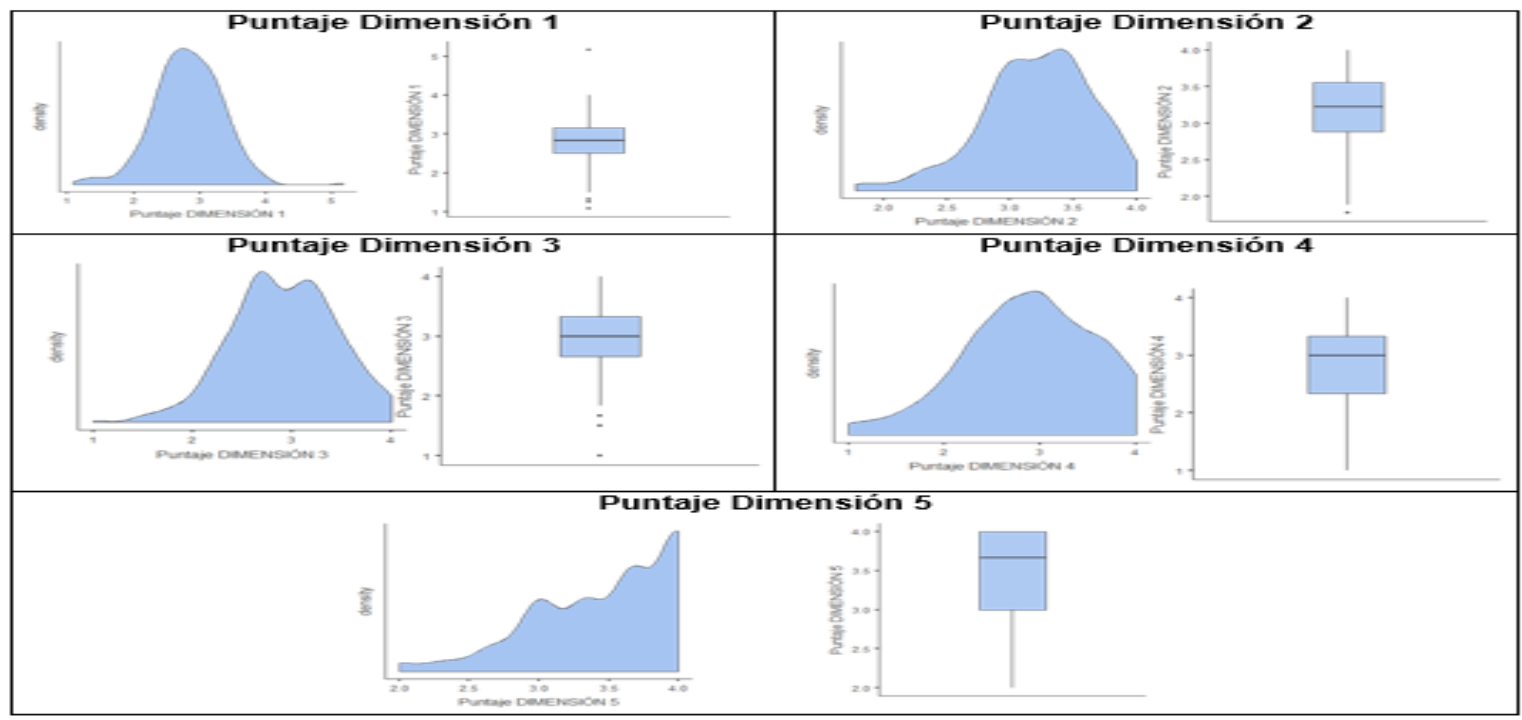

Fuente: Elaboración propia

Figura 2

Comparación jerárquica de dimensiones
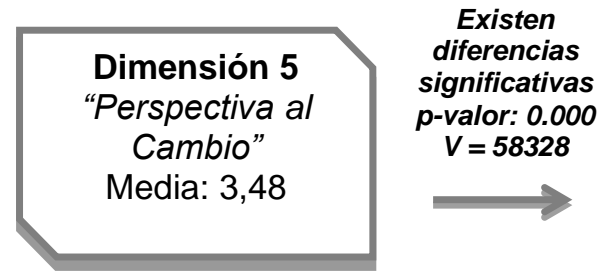

Existen significativas p-valor: 0.000 $V=58328$
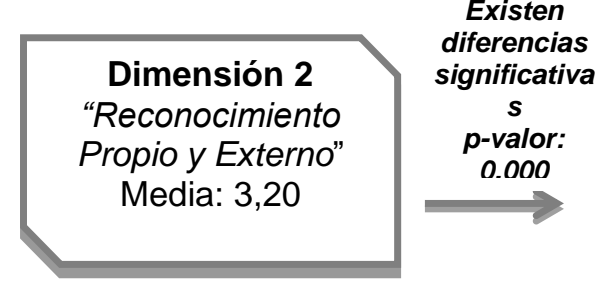

Dimensión 3

"Planificación

Estratégica"

Media: 2,92

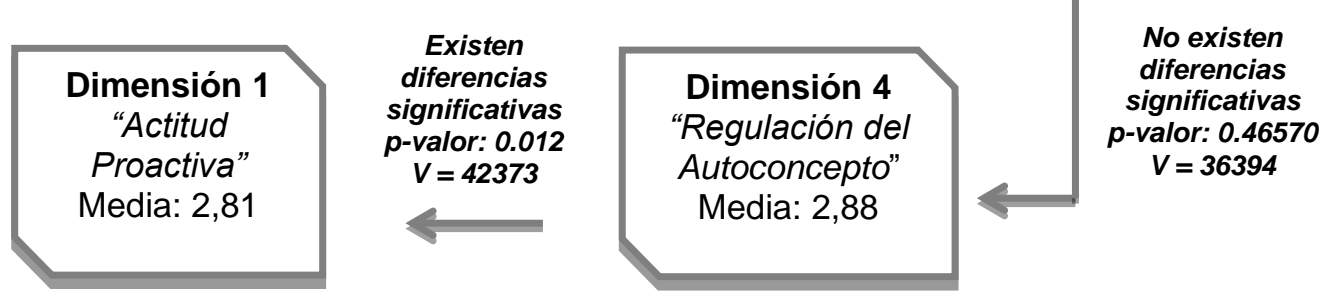

Fuente: Elaboración propia

En función de la dinámica de las puntuaciones observadas para el $3 \mathrm{Cl}$ se establecen cinco Niveles de creatividad e innovación. Es importante indicar que todos los niveles centrales poseen la misma amplitud, producto de que la distribución de las frecuencias de los niveles se comporta de manera normal (Test de Shapiro Wilk, $w=0,987$, $p$-valor=0,001). 
Autopercepción de las competencias de creatividad e innovación... Pamela Marcone-Dapelo et al.

Tabla 6

Niveles de desarrollo de creatividad e innovación según puntajes $3 \mathrm{Cl}$

\begin{tabular}{cc}
\hline Puntaje 3Cl & Nivel de Creatividad \\
\hline$[33 ; 60]$ & Estadio Experiencial \\
\hline$[60 ; 80]$ & Estadio Asociativo \\
\hline$[80 ; 100]$ & Estadio Productivo \\
\hline$[100 ; 120]$ & Estadio Social \\
\hline$[120 ; 132]$ & Estadio Innovador \\
\hline
\end{tabular}

Fuente: Elaboración propia, basado en Martínez, 2012 e Iglesias-Cortizas y Rodicio-García, 2013

Es relevante señalar que existen muchas formas de configurar un mismo puntaje, considerando pertinente definir la orientación de cada nivel de creatividad, de acuerdo con la siguiente Tabla:

\section{Tabla 7}

Orientación de la creatividad e innovación según niveles de desarrollo

\begin{tabular}{ccc}
\hline Puntaje & $\begin{array}{c}\text { Configuración del puntaje según } \\
\text { dimensión }\end{array}$ & Orientación \\
\hline$[0 ; 10]$ & Actitud Proactiva & Proactiva \\
\hline$[10 ; 13,5]$ & Reconocimiento Propio y Externo & Analítica \\
\hline$[13,5 ; 16,5]$ & Planificación Estratégica & Planificadora \\
\hline$[16,5 ; 20]$ & Regulación del Autoconcepto & Reguladora \\
\hline$[20 ; 25]$ & Perspectiva al Cambio & Progresista \\
\hline
\end{tabular}

Fuente: Elaboración propia

\section{Proceso analítico para establecer las orientaciones}

Para establecer la orientación de la creatividad e innovación, se han definido algunos elementos cuantitativos formales a describir:

Sean $F_{p}(A)=\frac{1}{N_{A}}, F_{p}(B)=\frac{1}{N_{B}}, F_{p}(C)=\frac{1}{N_{C}}, F_{p}(D)=\frac{1}{N_{D}}$ y $F_{p}(E)=\frac{1}{N_{E}}$ los respectivos factores de ponderación de cada una de las dimensiones. Donde $\mathrm{N}_{A}, \mathrm{~N}_{B}, \mathrm{~N}_{\mathrm{C}}, \mathrm{N}_{\mathrm{D}}$ y $\mathrm{N}_{\mathrm{E}}$ corresponden a las puntuaciones máximas posibles de obtener en cada una de las respectivas dimensiones.

Sean $\mathrm{n}_{\mathrm{A}}, \mathrm{n}_{\mathrm{B}}, \mathrm{n}_{\mathrm{C}}, \mathrm{n}_{\mathrm{D}}$ y $\mathrm{n}_{\mathrm{E}}$, las respectivas puntuaciones obtenidas en cada una de las dimensiones, luego la orientación de la creatividad e innovación quedará definida por:

$$
\mathrm{o}_{\mathrm{p}}=\max \left\{\frac{\mathrm{n}_{\mathrm{A}}}{\mathrm{N}_{\mathrm{A}}}, \frac{\mathrm{n}_{\mathrm{B}}}{\mathrm{N}_{\mathrm{B}}}, \frac{\mathrm{n}_{\mathrm{C}}}{\mathrm{N}_{\mathrm{C}}}, \frac{\mathrm{n}_{\mathrm{D}}}{\mathrm{N}_{D}}, \frac{\mathrm{n}_{\mathrm{E}}}{\mathrm{N}_{\mathrm{E}}}\right\}
$$

Si $\mathrm{O}_{\mathrm{p}}=\frac{\mathrm{n}_{\mathrm{A}}}{\mathrm{N}_{\mathrm{A}}}$, entonces se dirá que la orientación es Proactiva, si $\mathrm{O}_{\mathrm{p}}=\frac{\mathrm{n}_{\mathrm{B}}}{\mathrm{N}_{\mathrm{B}}}$, entonces se dirá que la orientación es Analítica, si $\mathrm{O}_{\mathrm{p}}=\frac{\mathrm{n}_{\mathrm{C}}}{\mathrm{N}_{\mathrm{C}}}$, entonces se dirá que la orientación es Planificadora, si $\mathrm{O}_{\mathrm{p}}=\frac{\mathrm{n}_{\mathrm{D}}}{\mathrm{N}_{\mathrm{D}}}$, entonces se dirá que la orientación es Reguladora y si $\mathrm{O}_{\mathrm{p}}=\frac{\mathrm{nE}_{\mathrm{E}}}{\mathrm{N}_{\mathrm{E}}}$, entonces se dirá que la orientación es Progresista. 
Autopercepción de las competencias de creatividad e innovación... Pamela Marcone-Dapelo et al.

En el caso de que dos o más componentes de $0_{\mathrm{p}}$ se identifiquen con el máximo, se dirá que la orientación es compuesta.

Es así, que basados en el cruce de Niveles de creatividad e innovación y las orientaciones correspondientes, se obtiene la siguiente información:

\section{Tabla 8}

Distribución de las orientaciones de la muestra, en función del Nivel de creatividad alcanzado

\begin{tabular}{lccccc}
\hline Orientación / Nivel & Proactiva & Analítica & Planificadora & Reguladora & Progresista \\
\hline $\begin{array}{l}\text { Estadio } \\
\text { Experiencial }\end{array}$ & 3 & 0 & 0 & 0 & 0 \\
\hline Estadio Asociativo & 4 & 19 & 0 & 0 & 0 \\
\hline Estadio Productivo & 0 & 36 & 144 & 0 & 0 \\
\hline Estadio Social & 0 & 0 & 92 & 92 & 0 \\
\hline Estadio Innovador & 0 & 0 & 0 & 22 & 1
\end{tabular}

Fuente: Elaboración propia

Se puede apreciar en la Tabla 8 que, a mayor nivel de desarrollo de la creatividad, es evidente el predominio en las orientaciones planificadora y reguladora, siendo estas el equivalente al $79.4 \%$ de la muestra. Mientras que en niveles inferiores se observan orientaciones tendientes a la proactividad y analítica.

Una vez establecidas las orientaciones y niveles de creatividad e innovación de los sujetos de la muestra, se procede a generar el análisis de otras variables relacionadas, las que se describen a continuación.

En primera instancia se analizan las variables intrínsecas de los sujetos de la muestra, y posteriormente las variables extrínsecas, de orden académico.

\section{Tabla 9}

Caracterización de las variables de acuerdo con el nivel de creatividad e innovación

\begin{tabular}{|c|c|c|c|c|c|c|}
\hline \multicolumn{2}{|c|}{ Nivel /Variable } & $\begin{array}{l}\text { Estadio } \\
\text { Innovador }\end{array}$ & $\begin{array}{l}\text { Estadio } \\
\text { Social }\end{array}$ & $\begin{array}{l}\text { Estadio } \\
\text { Productivo }\end{array}$ & $\begin{array}{l}\text { Estadio } \\
\text { Asociativo }\end{array}$ & $\begin{array}{l}\text { Estadio } \\
\text { Experiencial }\end{array}$ \\
\hline \multirow[t]{2}{*}{ Sexo } & Femenino & $18(5,8 \%)$ & $138(44,8 \%)$ & $133(43,2 \%)$ & $16(5,2 \%)$ & $3(1 \%)$ \\
\hline & Masculino & $5(4,8 \%)$ & 46 (43,8 \%) & $47(44,8 \%)$ & $7(6,6 \%)$ & 0 \\
\hline \multirow[t]{5}{*}{ Edad } & R1 [18; 22] & $12(5,04 \%)$ & $100(42 \%)$ & $108(45,4 \%)$ & $17(07,14 \%)$ & $1(0,42 \%)$ \\
\hline & $\mathrm{R} 2$ [22; 26] & $6(4,25 \%)$ & $67(47,52 \%)$ & $61(43,26 \%)$ & $6(4,26 \%)$ & $1(0,71 \%)$ \\
\hline & R3 [26; 30] & $2(1,41 \%)$ & $14(51,85 \%)$ & $11(40,74 \%)$ & 0 & 0 \\
\hline & R4 [30; 34] & $2(40 \%)$ & $3(60 \%)$ & 0 & 0 & 0 \\
\hline & R5 [34; 38] & $1(50 \%)$ & 0 & 0 & 0 & $1(50 \%)$ \\
\hline
\end{tabular}

Fuente: Elaboración propia 
Autopercepción de las competencias de creatividad e innovación... Pamela Marcone-Dapelo et al.

Tabla 9 (Continuación)

Caracterización de las variables de acuerdo con el nivel de creatividad e innovación

\begin{tabular}{|c|c|c|c|c|c|c|}
\hline \multicolumn{2}{|c|}{ Nivel /Variable } & $\begin{array}{l}\text { Estadio } \\
\text { Innovador }\end{array}$ & $\begin{array}{l}\text { Estadio } \\
\text { Social }\end{array}$ & $\begin{array}{l}\text { Estadio } \\
\text { Productivo }\end{array}$ & $\begin{array}{l}\text { Estadio } \\
\text { Asociativo }\end{array}$ & $\begin{array}{l}\text { Estadio } \\
\text { Experiencial }\end{array}$ \\
\hline \multirow[t]{3}{*}{ Ciclo } & $\begin{array}{l}\text { C1, Ciclo } \\
\text { Bachillerato }\end{array}$ & $8(4 \%)$ & $94(47,5 \%)$ & $83(42 \%)$ & $11(6 \%)$ & $1(0,50 \%)$ \\
\hline & $\begin{array}{l}\text { C2, Ciclo } \\
\text { Licenciatura }\end{array}$ & $12(6,5 \%)$ & $74(39,8 \%)$ & $87(46,8 \%)$ & $11(5,9 \%)$ & $2(1 \%)$ \\
\hline & $\begin{array}{l}\text { C3, Ciclo de } \\
\text { Síntesis } \\
\text { Profesional }\end{array}$ & $3(10,34 \%)$ & $15(51,72 \%)$ & $10(34,5 \%)$ & $1(3,44 \%)$ & 0 \\
\hline \multirow[t]{5}{*}{ Carrera } & Kinesiología & $8(6,06 \%)$ & $69(52,2 \%)$ & $50(37,87 \%)$ & $4(3,03 \%)$ & $1(0,75 \%)$ \\
\hline & $\begin{array}{l}\text { Terapia } \\
\text { Ocupacional }\end{array}$ & $4(5,71 \%)$ & $29(41,42 \%)$ & $32(45,71 \%)$ & $5(7,1 \%)$ & 0 \\
\hline & Nutrición & $2(3,13 \%)$ & $25(39,06 \%)$ & $34(53,13 \%)$ & $3(4,68 \%)$ & 0 \\
\hline & Enfermería & $1(1,53 \%)$ & $27(41,53 \%)$ & $28(43,07 \%)$ & $7(10,76 \%)$ & $1(1,5 \%)$ \\
\hline & Fonoaudiología & $8(9,75 \%)$ & $33(40,24 \%)$ & $36(43,90 \%)$ & $4(4,87 \%)$ & $1(1,2 \%)$ \\
\hline
\end{tabular}

Fuente: Elaboración propia

Niveles de las competencias creativas e innovadoras $\mathrm{v} / \mathrm{s}$ variables intrínsecas

En cuanto a los Niveles de creatividad e innovación se evidencia una tendencia común entre hombre y mujeres, concentrándose los dos grupos en los Niveles de creatividad e innovación de "Estadio Social" (44,8\% de las mujeres y $43,8 \%$ de los hombres) y "Estadio productivo" (43,2\% de las mujeres y $44,8 \%$ de los hombres).

De la información recabada es posible inferir que no existe relación estadísticamente significativa entre el sexo y el nivel de creatividad (test de Pearson: Chi Cuadrado $=1.5543$, con un p-valor de 0.817).

Respecto de la relación edad v/s nivel de creatividad se distribuye en los rangos de la siguiente forma: R1 principalmente nivel "Estadio productivo". R2 predominan los niveles "Estadio Productivo" y "Estadio Social". R3 y R4 mayoritariamente en "Estadio Social". R5 la distribución es equivalente entre "Estadio Experiencial" y "Estadio Innovador". Del total de la muestra en el R1 con Nivel "Estadio Productivo", se encuentra un $26.2 \%$ de los sujetos, seguido en el mismo rango por el nivel de "Estadio Social" correspondiente a un 24.2\% (Tabla 9).

Los datos evidencian que existe relación estadísticamente significativa entre el Rango de Edad y el Nivel de Creatividad (test de Pearson: Chi Cuadrado = 94.614, con un p-valor de 0.000). 
Autopercepción de las competencias de creatividad e innovación... Pamela Marcone-Dapelo et al.

Niveles de las competencias creativas e innovadoras $v / s$ variables extrínsecas

Aludiendo a la relación entre el Ciclo Real - Nivel de Creatividad, se establece que los Ciclos 1 y 2 concentran el $82 \%$ de la muestra en los Niveles de creatividad e innovación de "Estadio Productivo" y "Estadio Social".

Los datos indican que no existe relación estadísticamente significativa entre el Ciclo real que cursan los integrantes de la muestra y el nivel de Creatividad (test de Pearson: Chi Cuadrado = 5,9774 , con un p-valor de 0.649).

Es así que la relación entre la carrera y el nivel de creatividad se concentra entre los niveles de "Estadio Productivo" y "Estadio Social" con un 43.5\% y 44,3\% respectivamente (Tabla 9).

De los datos obtenidos es posible aseverar que no existe relación estadísticamente significativa entre la carrera que cursan los integrantes de la muestra y el nivel de Creatividad (test de Pearson: Chi Cuadrado $=17.345$, con un p-valor de $=0.363$ ).

\section{Tabla 10}

Caracterización de las variables de acuerdo con la orientación de la creatividad e innovación

\begin{tabular}{|c|c|c|c|c|c|c|}
\hline \multicolumn{2}{|c|}{$\begin{array}{l}\text { Orientación / } \\
\text { Variable }\end{array}$} & \multirow{2}{*}{$\begin{array}{l}\text { Analítica } \\
40(13 \%)\end{array}$} & \multirow{2}{*}{$\begin{array}{l}\text { Planificadora } \\
180(58,4 \%)\end{array}$} & \multirow{2}{*}{$\begin{array}{l}\text { Proactiva } \\
7(2,3 \%)\end{array}$} & \multirow{2}{*}{$\begin{array}{l}\text { Progresista } \\
1(0,3 \%)\end{array}$} & \multirow{2}{*}{$\begin{array}{l}\text { Reguladora } \\
80(26 \%)\end{array}$} \\
\hline Sexo & Femenino & & & & & \\
\hline & Masculino & $15(14,3 \%)$ & $56(53,3 \%)$ & 0 & 0 & $34(32,4 \%)$ \\
\hline \multirow[t]{5}{*}{ Edad } & R1 [18; 22] & $34(14,3 \%)$ & $146(61,3 \%)$ & $4(1,7 \%)$ & $1(0,4 \%)$ & $53(22,3 \%)$ \\
\hline & R2 [22; 26] & $19(13,5 \%)$ & $72(51,1 \%)$ & $2(1,4 \%)$ & 0 & 48 (34\%) \\
\hline & R3 [26; 30] & $2(7,4 \%)$ & $15(55,6 \%)$ & 0 & 0 & $10(37 \%)$ \\
\hline & R4 [30; 34] & 0 & $3(60 \%)$ & 0 & 0 & $2(40 \%)$ \\
\hline & R5 [34; 38] & 0 & 0 & $1(50 \%)$ & 0 & $1(50 \%)$ \\
\hline \multirow[t]{3}{*}{$\begin{array}{l}\text { Ciclo } \\
\text { Real }\end{array}$} & $\begin{array}{l}\text { C1 } \\
\text { Ciclo } \\
\text { Bachillerato }\end{array}$ & $29(14,65 \%)$ & $114(57,57 \%)$ & $2(1,01 \%)$ & $1(0,51 \%)$ & $52(26,26 \%)$ \\
\hline & $\begin{array}{l}\text { C2 } \\
\text { Ciclo } \\
\text { Licenciatura }\end{array}$ & $21(11,29 \%)$ & $109(58,6 \%)$ & $5(2,69 \%)$ & 0 & $51(27,42 \%)$ \\
\hline & $\begin{array}{l}\text { C3 } \\
\text { Ciclo de } \\
\text { Síntesis } \\
\text { Profesional }\end{array}$ & $5(17,3 \%)$ & $13(44,8 \%)$ & 0 & 0 & $11(37,90 \%)$ \\
\hline \multirow[t]{5}{*}{ Carrera } & Kinesiología & $15(11,4 \%)$ & 68 (51,5\%) & $1(0,8 \%)$ & 0 & $48(36,3 \%)$ \\
\hline & $\begin{array}{l}\text { Terapia } \\
\text { Ocupacional }\end{array}$ & $13(18,57 \%)$ & $40(57,14 \%)$ & 0 & 0 & $17(24,29 \%)$ \\
\hline & $\begin{array}{l}\text { Nutrición } \\
\text { dietética }\end{array}$ & $7(10,93 \%)$ & $41(64,6 \%)$ & $2(3,13 \%)$ & 0 & $14(21,88 \%)$ \\
\hline & Enfermería & $9(13,8 \%)$ & $44(67,7 \%)$ & $2(3,1 \%)$ & 0 & $10(15,4 \%)$ \\
\hline & Fonoaudiología & $11(13,41 \%)$ & $43(52,44 \%)$ & $2(2,44 \%)$ & $1(1,22 \%)$ & $25(30,49 \%)$ \\
\hline
\end{tabular}

Fuente: Elaboración propia 
Autopercepción de las competencias de creatividad e innovación... Pamela Marcone-Dapelo et al.

Orientación de las competencias creativas e innovadoras v/s variables intrínsecas

En cuanto a la relación sexo - orientación, es posible evidenciar que las orientaciones tanto en hombres, como mujeres siguen una misma tendencia de distribución porcentual, bajo el siguiente detalle:

Mujeres y hombres se distribuyen de forma similar, encontrando los mayores porcentajes en la "Orientación Planificadora", seguido de la "Orientación Reguladora". Basado en los datos obtenidos se puede aseverar que no existe relación entre el sexo y la orientación de la creatividad e innovación en los sujetos consultados (test de Pearson: Chi Cuadrado $=4.3485$, con un $p$-valor de 0.3609).

La relación edad v/s orientación se distribuye en los rangos de la siguiente forma: Rangos 1 a 4: predominio "Planificador" y en el Rango 5 la distribución es equivalente entre "Proactivo" y "Regulador". Del total de la muestra en el R1 con Orientación Planificadora, se encuentra un $35,4 \%$ de los sujetos. Seguido de R2 con Orientación Planificadora correspondiente a un 17.4\% (Tabla 10).

Los datos indican que existe relación estadísticamente significativa entre el Rango de Edad y la orientación de la creatividad e innovación (test de Pearson: Chi Cuadrado $=39.579$, con un pvalor de 0.000).

\section{Orientación de las competencias creativas e innovadoras v/s variables extrínsecas}

En cuanto a la relación de los Ciclos - Orientación, se encuentra que en los Ciclos 1 y 2 el predominio de orientación es "Planificadora" con $27.6 \%$ y $26.4 \%$ de la muestra total, respectivamente.

De los datos se infiere que no existe relación estadísticamente significativa entre el Ciclo real que cursan los integrantes de la muestra y la orientación de la creatividad e innovación (test de Pearson: Chi Cuadrado $=6.4771$, con un p-valor de 0.594).

Respecto a la relación entre las carreras que cursan los integrantes de la muestra, frente a la orientación de la creatividad e innovación, se aprecia un predominio de la orientación "Planificadora" en todas las carreras, lo que corresponde al $57 \%$ de la unidad muestral (Tabla 10).

Con base en los datos, se puede inferir que no existe relación estadísticamente significativa entre las carreras que cursan los integrantes de la muestra y la orientación de la creatividad e innovación (test Pearson: Chi Cuadrado = 21.12, con un p-valor de 0.174).

\section{Conclusiones y Discusión}

La universidad es la institución encargada de proveer al alumnado de nuevos aprendizajes, entregarles competencias del ser, saber y hacer, que no pueden seguir siendo la tradicional forma 
de conocimiento por reproducción (Rodríguez et al., 2018). Por lo que la educación universitaria debe evolucionar desarrollando competencias sociales, específicamente competencias creativas y así responder a los nuevos desafíos de la sociedad (Chanal, 2019). A su vez, el éxito y el fracaso académico, según Fernández Enguita, Mena y Riviere, se relacionan con las características personales y sociales de los estudiantes (Mudarra et al., 2016). Siendo la creatividad e innovación claves en el desarrollo del aprendizaje universitario (Raso, 2019) y elementos clave para obtener ventaja competitiva en todos los campos de conocimiento, inserción laboral y en el trascurso de la vida en la sociedad (Hernández et al., 2015; Raso, 2019).

Dada la relevancia de identificar el desarrollo de la creatividad e innovación, este estudio aporta una adaptación para Chile del instrumento $3 \mathrm{Cl}$, brindando a la comunidad educativa un elemento fiable, con alta consistencia interna y válido. Conformado, a través del análisis factorial por cinco dimensiones, en las que se conservan los 33 reactores del instrumento original.

Basada en el análisis de los resultados, esta investigación propone una alternativa para indagar la autopercepción de la creatividad y la innovación en estudiantes universitarios de las ciencias de la salud, permitiendo establecer su nivel de desarrollo, y su orientación, es decir, la tendencia del enfoque de dichas competencias. En este estudio, se encontró un predominio del nivel de desarrollo correspondiente al estadio productivo y a la orientación planificadora, seguido del nivel de desarrollo del estadio social, con orientación reguladora.

En cuanto a las dimensiones, se evidenciaron diferencias estadísticamente significativas entre ellas, salvo en la comparación entre las dimensiones "Planificación estratégica" y "Regulación del Autoconcepto", las cuales incluso podrían agruparse como una sola dimensión ( $p$-valor: 0.46570 ). Esto puede explicarse porque tanto la planificación, como la regulación son habilidades que se agrupan en las funciones ejecutivas, correspondientes a funciones mentales superiores autorreguladas, esenciales para el desarrollo y mediadas por el aprendizaje (Bernal-Ruiz et al., 2018), que permiten al individuo ejercitar el autocontrol de la conducta, manejar la información mentalmente, resolver problemas y adaptarse flexiblemente a situaciones inesperadas. Por tanto, se trata de procesos que constituyen un papel fundamental en el éxito cognitivo, académico, emocional, comportamental y social (Pardos et al., 2018).

Respecto del análisis efectuado entre los factores intrínsecos y extrínsecos con la creatividad e innovación, se pudo establecer que solo existe relación estadísticamente significativa con la variable intrínseca edad, lo cual es coincidente con lo presentado por Caballero et al (2018), quien indica un comportamiento significativamente diverso en un grupo de niños de edad superior versus otros grupos etarios.

Dentro de las limitantes del estudio, se encuentra que la distribución de la muestra no es homogénea en cuanto a la cantidad de estudiantes por carreras y ciclos de formación. Por lo demás, las carreras de la FCS de la UPLA, cuentan con un nivel de consolidación diferente respecto de sus procesos de innovación curricular, lo que podría impactar en el abordaje de la creatividad e innovación en su proceso formativo.

Si bien este estudio se centra en estudiantes universitarios del área de la salud, las competencias de creatividad e innovación son transversales a todos los profesionales, ya que, no solo le serán útiles en su vida profesional, sino también personal (Chanal, 2019). Siendo clave el diagnóstico de la autopercepción de los niveles y orientaciones de creatividad e innovación en la formación de todos los profesionales (Felek et al., 2019, De la Iglesia, 2018).

El diagnóstico de la autopercepción de los niveles y orientaciones de creatividad e innovación contribuye a la elección de ambientes de aprendizaje y prácticas docentes según niveles hallados, desarrollando, no solo estas competencias, sino también otras habilidades personales como es la inteligencia emocional (Rodríguez, 2018)

Es importante destacar que existen programas de intervención de creatividad e innovación en todos los niveles de formación de grado, diferenciados según los niveles de creatividad y sus 
orientaciones, como el estudio realizado por Cabello et al. (2019), obteniendo resultados de potenciamiento de estas competencias. Así como lo planteado por Chanal (2019) y Tican et al., (2019) quienes aluden a que la enseñanza práctica logra mejores resultados en el desarrollo de la comunicación, pensamiento crítico, colaboración y aprendizaje creativo, promoviendo el trabajo en equipos transdisciplinares.

En virtud de lo expuesto, se hace necesario ampliar este estudio a otras realidades universitarias y asociarlo a otros aspectos socioculturales, para así establecer si los resultados que se obtengan aportan, con las adecuaciones curriculares que se hacen sistemáticamente bajo el modelo educativo institucional, de manera que la creatividad ya no tenga un espacio limitado en el sistema de educación chileno (Pllana, 2019), recalcando que es responsabilidad de la universidad generar ambientes creativos para el individuo o comunidad en general (Nimer, 2016), incluyendo en los programas de educación técnica y/o profesional competencias blandas que fortalezcan las capacidades laborales de los egresados (Rueda, 2020).

\section{Referencias bibliográficas}

Arias, C., Giraldo, D. y Anaya, L. (2013). Competencia creatividad e innovación: conceptualización y abordaje en la educación. Katharsis: Revista de Ciencias Sociales 15, 195-214.

Bermejo, R., Hernández, D., Ferrando, M., Soto, G., Sainz, M. y Prieto, M. (2010). Creatividad, inteligencia sintética y alta habilidad. Revista electrónica interuniversitaria de formación del profesorado, 13(1), 97-109.

Bernal-Ruiz, F., Rodríguez-Vera, M., González-Campos, J. y Torres-Álvarez, A. (2018). Competencias parentales que favorecen el desarrollo de funciones ejecutivas en escolares. Revista Latinoamericana de Ciencias Sociales, Niñez y Juventud, 16(1), 163176. https://doi.org/10.11600/1692715x.16109

Caballero, Á. y Fernández, M. (2018). Creatividad y rendimiento académico: un estudio de caso con alumnos de $4^{\circ}$ curso de educación secundaria. Revista Iberoamericana de Educación, 78(2), 77-95.

Caballero, P. Á., Ruiz, S. S. y Almagro, M. L. B. (2019). Analysis of creativity among university students. Differences depending on gender, age, and choice of studies. Educacion XXI, 22(2), 213-234. https://doi.org/10.5944/educxx1.22552

Chanal, V. (2019). La creatividad de la enseñanza en la educación superior: una perspectiva comunitaria. Aula Abierta, 48(4), 407. https://doi.org/10.17811/rifie.48.4.2019.407-418

Chen, K., Monrouxe, L., Lu, Y., Jenq, C., Chang, Y., Chang, Y. y Chai, P. (2018). Academic outcomes of flipped classroom learning: a meta-analysis. Medical Education 52(9), 910 924. https://doi.org/10.1111/medu.13616

Chiecher, A.-C., Elisondo, R.-C., Paoloni, P.-V. y Donolo, D.-S. (2018). Creatividad, género y rendimiento académico en ingresantes de ingeniería. Revista iberoamericana de educación superior, 9(24), 138-151. https://doi.org/10.22201/iisue.20072872e.2018.24.3365 
Autopercepción de las competencias de creatividad e innovación... Pamela Marcone-Dapelo et al.

De la Iglesia, M. C. (2018). Learning based on a teaching project: Learning, creativity, innovation and new roles of the teacher's training in the digital age. Revista Complutense de Educacion, 29(4), 1253-1278. https://doi.org/10.5209/RCED.55256

Felek, S. Ö. y Gül, Ö. (2019). Evaluation of Strategies of Creativity Development Used in Store Design Projects Based on Student Projects. Design and Technology Education, 24(1), 121. https://ojs.Iboro.ac.uk/DATE/article/view/2517

Fernández, I., Eizaguirre, A., Arandia, M., Ruiz, P. y Ezeiza, A. (2012). Creatividad e innovación: claves para intervenir en contextos de aprendizaje. REICE: Revista Electrónica Iberoamericana sobre Calidad, Eficacia y Cambio en Educación, 10(2), 23-40.

González, J., Carvajal, C. y Viveros, F. (2016). Coeficientes edumétricos para la validez y dificultad de un test: Propuesta. Estudios pedagógicos, 42(3), 467-481. https://doi.org/10.4067/S0718-07052016000400025

Hernández, I., Alvarado, J. y Luna, S. (2015). Creatividad e innovación: competencias genéricas o transversales en la formación profesional. Revista Virtual Universidad Católica del Norte, 1(44), 135-151.

Hernández, R., Fernández, C. y Batista, P. (2014). Metodología de la investigación (6ª ed.). Editorial McGraw-Will.

Iglesias-Cortizas, M. y Rodicio-García, M. (2013 ). El desarrollo de la creatividad e innovación. Un reto ante la crisis actual. Revista de Investigación en Educación, 11 (1) 134-148.

Jiménez, Y., Hernández, J. y González, M. (2013). Competencias profesionales en la educación superior: justificación, evaluación y análisis. Innovación educativa, 13(61), 45-65.

Jiménez, Y., Gonzalez, M., y Ortega, J. (2014). Evaluación por competencias. Entre la tradición y el cambio. Revista Iberoamericana de Producción Académica y Gestión Educativa, 1(2). https://www.pag.org.mx/index.php/PAG/article/download/192/240

Marcone-Dapelo, P., Godoy-Briceño, J., y Álvarez-Silva, D. (2018). Análisis Psicométrico de la Adaptación Chilena del Cuestionario de Competencias Creativas e Innovadoras $(3 \mathrm{Cl})$. Revista de Orientación Educacional, 32(62), 60-76.

Mudarra, M. J. y Garcia-Salguero, B. (2016). Habilidades sociales y éxito académico: expectativas de los profesores de educación secundaria. Revista Española de Orientación y Psicopedagogía, 27(1), 114-133. https://doi.org/10.5944/reop.vol.27.num.1.2016.17032

Namakforoosh, M. N. (2000). Metodología de la investigación. Editorial Limusa.

Oliveira, E., Almeida, L., Ferrándiz, C., Ferrando, M., Sainz, M. y Prieto, M. (2009). Tests de pensamiento creativo de Torrance (TTCT): elementos para la validez de constructo en adolescentes portugueses. Psicothema, 21(4), 562-567.

Neri, J. C. y Hernández, C. A. (2019). Los jóvenes universitarios de ingeniería y su percepción sobre las competencias blandas. RIDE. Revista Iberoamericana Para La Investigación y EI Desarrollo Educativo, 9(18), 768-791. https://doi.org/10.23913/ride.v9i18.445

Nimer, A. M. (2016). The Extent of the Contribution of the Educational Environment of Najran University in Developing Creative and Innovative Enterprises among the University Students. Journal of Educational Issues, 2(1), 305. https://doi.org/10.5296/jei.v2i1.9445

Pardos, A. y González, M. (2018). Intervención sobre las Funciones Ejecutivas (FE) desde el contexto educativo. Revista Iberoamericana de Educación, 78(1), 27-42. https://doi.org/10.35362/rie7813269 
Autopercepción de las competencias de creatividad e innovación... Pamela Marcone-Dapelo et al.

Paz, L., Hernández, E. y Van de Water, H. (2016). Los retos de la Educación Superior en el Siglo XXI. Revista Conrado, 12(55),17-24.

Pinilla, A. (2018). Educación en ciencias de la salud y en educación médica. Acta Médica Colombiana, 43(2), 61-65.

Pinilla-Roa, A. (2012). Aproximación conceptual a las competencias profesionales en ciencias de la salud. Revista de Salud Pública, 14(5), 852-864

Pllana, D. (2019). Creativity in Modern Education. World Journal of Education, 9(2), 136. https://doi.org/10.5430/wje.v9n2p136

Raso, F. (2019). Percepciones del Futuro Pedagogo sobre la Metodología de Enseñanza de la Creatividad. Revisión de la literatura. 17(1), 73-88.

Rodríguez, N. E., Delgadillo, M. D. y Torres, S. L. (2018). Los ambientes de aprendizaje constructivistas como alternativa para generar innovación en la universidad. International Journal of Information Systems and Software Engineering for Big Companies (IJISEBC), 5(2), 41-52.

Rueda, J. F. y Portilla, S. A. (2020). Training in general occupational skills, a challenge for higher education. I+D Revista de Investigaciones, 15(1), 37-44.

Steers, J. (2009). Creativity: Delusions, Realities, Opportunities and Challenges. Journal of Art and Design Education, 28(2), 126-138. https://doi.org/10.1111/j.1476-8070.2009.01600.x

Tena, M. (2010). Aprendizaje de la Competencia Creatividad e Innovación en el marco de una titulación adaptada al Espacio Europeo de Educación Superior. Formación universitaria, 3(2), 11-20. https://doi.org/10.4067/S0718-50062010000200003

Tican, C. y Deniz, S. (2019). Pre-service teachers' opinions about the use of 21 st century learner and 21 st century teacher skills. European Journal of Educational Research, 8(1), 181-197. https://doi.org/10.12973/eu-jer.8.1.181

Trujillo-Segoviano, J. (2014). El enfoque en competencias y la mejora de la educación. Ra Ximhai, 10(5), 307-322.

Universidad de Playa Ancha. (2017). Itinerario base de inovacion curricular. http://www.upla.cl/innovacioncurricular/wp-content/uploads/2012/06/ltinerario-baseinovacion-curricular2017.pdf

Valera, R. (2010). El proceso de formación del profesional en la educación superior basado en competencias: el desafío de su calidad, en busca de una mayor integralidad de los egresados. Civilizar Ciencias Sociales y Humanas, 10(18), 117-134.

Velásquez, B., Remolina de Cleves, N. y Calle Márquez, M. (2010). La creatividad como práctica para el desarrollo del cerebro total. Tabula Rasa(13),321-338.

Fecha de entrada: 17 de abril 2019

Fecha de revisión: 21 de mayo 2020

Fecha de aceptación: 21 de junio 2020 